\title{
Conseqüências de medicação em unidades de terapia intensiva e semi-intensiva
}

\author{
CONSEQUENCES OF MEDICAL ERRORS IN INTENSIVE AND SEMI-INTENSIVE CARE UNITS \\ CONSECUENCIAS DE LOS ERRORES DE MEDICACIÒN EN UNIDADES DE \\ CUIDADOS INTENSIVOS - SEMI INTENSIVOS.
}

\author{
Maria Cecília Toffoletto', Kátia Grillo Padilha²
}

\section{RESUMO}

O estudo objetivou caracterizar erros de medicação e avaliar conseqüências na gravidade dos pacientes e carga de trabalho de enfermagem em duas Unidades de Terapia Intensiva (UTI) e duas Semi-Intensiva (USI) de duas instituições hospitalares do município de São Paulo. A amostra foi constituída por 50 pacientes e os dados obtidos por meio do registro de ocorrências e prontuários, retrospectivamente. A gravidade e carga de trabalho de enfermagem foram avaliadas antes e após o erro. Do total de 52 erros, $12(23,08 \%)$ ocorreram por omissão de dose, $11(21,15 \%)$ e 9 $(17,31 \%)$ por medicamento e dose erradas, respectivamente. Não houve mudança na gravidade dos pacientes $(\mathrm{p}=0,316)$, porém houve aumento na carga de trabalho de enfermagem $(p=0,009)$. Quanto ao grupo de medicamentos envolvidos, potencialmente perigosos e não potencialmente perigosos, não houve diferenças estatisticamente significantes na gravidade $(\mathrm{p}=0,456)$ e na carga de trabalho de enfermagem $(p=0,264)$, após o erro de medicação.

\section{DESCRITORES}

Erros de medicação.

Unidades de Terapia Intensiva.

Carga de trabalho.

\section{ABSTRACT}

This report was aimed at characterizing medication errors and evaluating their consequences for the patients' conditions and for the nursing workload in the Intensive Care Units (ICU) and SemiIntensive Care Units (SICU) of two hospitals in the city of São Paulo. The sample was 50 patients, and data was gathered in record logs. The severity of the conditions and the nursing workload were assessed before and after the occurrence. Out of a total of 52 medication errors, 12 $(23.80 \%)$, were non-administration of dosage, $11(21.15 \%)$ were wrong medication, and 9 (17.31\%) excessive dosage. There were no changes in patient conditions $(p=0.316)$, but the nursing workload increased $(\mathrm{p}=0.009)$. As for the medication group, i.e, potentially dangerous or non-dangerous, there were no statistically significant differences either in the severity of the patients $(p=0.456)$ or in the nursing workload $(\mathrm{p}=0.264)$ after the occurrence.

\section{KEY WORDS}

Medication errors.

Intensive Care Units.

Workload.

\section{RESUMEN}

En el presente estudio se tuvo como objetivo caracterizar errores de medicación y evaluar consecuencias en la gravedad de los pacientes y carga de trabajo de enfermería en dos Unidades de cuidados Intensivos (UCI) y dos Semi-Intensivos (USI) de dos instituciones hospitalarias del municipio de Sao Paulo. La muestra constituida por 50 pacientes fueron obtenidos por medio del registro de ocurrencias e historias clínicas, retrospectivamente. La gravedad y carga de trabajo de enfermería fueron evaluadas antes y después del error. Del total de 52 errores, 12 (23,08\%) ocurrieron por omisión deladosis, $11(21,15 \%)$ y $9(17,31 \%)$ por medicamento y dosis erradas, respectivamente. No se observó cambio en la gravedad de los pacientes $(p=0,316)$, no obstante hubo aumento en la carga de trabajo de enfermería $(p=0,009)$. En cuanto al grupo de medicamentos involucrados, potencial y no potencialmente peligrosos, no existieron diferencias estadísticamente significativas en la gravedad $(p=0,456)$ y en la carga de trabajo de enfermería $(p=0,264)$, después del error de medicación.

\section{DESCRIPTORES}

Errores de medicación.

Unidades de Cuidado Intensivo.

Carga de trabajo.

\footnotetext{
1 Enfermeira. Mestranda do Programa de Pós-Graduação na

Saúde do Adulto da Escola de Enfermagem da Universidade de São Paulo (EEUSP) mariacel@usp.br 2 Professora Doutora do Departamento de Enfermagem MédicoCirúrgica da EEUSP kgpadilha@usp.br
} 


\section{INTRODUÇÃO}

A segurança dos pacientes no decorrer da internação hospitalar, têm merecido atenção crescente dos enfermeiros na busca por uma assistência que assegure um máximo de qualidade e um mínimo de riscos para o cliente. Nesse sentido, no que se refere à administração de medicamentos, a ocorrência de erros durante qualquer etapa desse processo, não só é indesejável para o alcance da qualidade dos serviços, como prejudicial para o paciente, equipe multidisciplinar e instituição hospitalar ${ }^{(1-6)}$.

As perdas de natureza emocional, física e financeira da equipe, família, instituição e sociedade são consideráveis. No entanto, as repercussões para os pacientes são as mais preocupantes, uma vez que podem agravar suas condições clínicas e causar injúrias temporárias, permanentes e até a morte $^{(3)}$. Por outro lado, também o profissional de enfermagem sofre as conseqüências desse tipo de evento, quer pelas sanções administrativas e legais possíveis, quer pela sobrecarga de trabalho a que fica exposto pela intensificação dos controles e vigilância do paciente.

Nas Unidades de Terapia Intensiva (UTI) e Unidades Semi Intensiva (USI), a abordagem dos erros de medicação e suas conseqüências para o paciente e equipe de enfermagem merecem enfoque particular. A complexa terapia medicamentosa, o uso de inúmeros medicamentos potencialmente perigosos (MPP) associados à gravidade e instabilidade clínica dos pacientes, justificam uma análise focalizada, pois, nessas circunstâncias, as conseqüências podem ser mais danosas. No entanto, na prática assistencial de enfermagem em UTI e USI, onde a administração de medicamentos é uma das atividades de maior importância, esse tipo de evento ocorre e nem sempre é valorizado pela equipe de enfermagem. Observa-se com freqüência a tendência a desconsiderá-los por se acreditar que, na maioria das vezes, não acarretam repercussões mais graves para os pacientes.

Além dos problemas inerentes à prática assistencial, verifica-se na literatura uma carência de estudos que focalizem objetivamente as conseqüências dos erros de medicação nas condições clínicas do paciente grave e na carga de trabalho da equipe de enfermagem com o uso de instrumentos de medida objetivos, validados e reconhecidos internacionalmente, o que se tornou possível, no entanto, com o desenvolvimento dos diferentes índices atualmente disponíveis.

Nesse sentido, os instrumentos Simplified Acute Physiology Score (SAPS) e o Therapeutic Intervention Scoring System (TISS) têm se mostrado adequados respectivamente, para a caracterização da gravidade das condições clínicas, tendo por base as condições fisiológicas do paciente, e para a avaliação da carga de trabalho de enfermagem, segundo as intervenções terapêuticas realizadas ${ }^{(7-9)}$. Por serem versões simplificadas, além de oferecerem facilidade na coleta de dados, indispensáveis diante do dinamismo de uma UTI, permitem sua utilização também nas Unidades de Cuidados Intermediários ${ }^{(10-12)}$.

Verifica-se, portanto, que tais instrumentos, por expressarem numericamente a gravidade dos doentes e as necessidades de cuidados de enfermagem, permitem também estimar as conseqüências dos erros de medicação nas condições clínicas do paciente e na carga de trabalho na UTI e USI.

Diante do interesse em avaliar objetivamente as conseqüências dos erros de medicação em UTI e USI, com a finalidade de oferecer subsídios para a prevenção desses eventos e para a implementação de um processo sistematizado para a sua monitorização, propõe-se a realização do presente estudo, com os seguintes objetivos: identificar os erros de medicação ocorridos com os pacientes durante a internação em UTI e USI quanto ao tipo, horário da ocorrência e grupo de medicamentos envolvidos; verificar alterações na gravidade dos pacientes e na carga de trabalho de enfermagem após a ocorrência de erro de medicação, segundo SAPS II e TISS 28 respectivamente; verificar alterações na gravidade do paciente e na carga de trabalho de enfermagem após a ocorrência de erro de medicação, segundo o grupo de medicamentos envolvidos.

\section{CASUÍSTICA E MÉTODO}

Trata-se de um estudo com abordagem quantitativa, descritivo-comparativo, retrospectivo, desenvolvido em duas UTIs e USIs de dois hospitais do Município de São Paulo, um privado e um público.

A amostra foi composta por 50 pacientes, sendo 40 da instituição privada e dez da pública, admitidos nas UTIs e USIs no período compreendido entre os anos de 2000 e 2003, submetidos a tratamento clínico e/ou cirúrgico que sofreram algum tipo de erro de medicação durante a permanência nessas unidades.

Após autorização das Comissões de Ética e Pesquisa, os dados referentes à identificação pessoal dos pacientes, da internação, das condições de gravidade e da carga de trabalho de enfermagem, 24 horas antes e 24 horas após o erro, foram coletados por meio dos registros de ocorrências de cada instituição, seguido da consulta aos prontuários dos pacientes vítimas de erros de medicação.

Para a caracterização da amostra de pacientes e dos erros de medicação segundo o tipo, horário e grupo de medicamentos, foi utilizada a estatística descritiva. O teste tpareado foi usado para análise das alterações da gravidade dos pacientes (SAPS II) e da carga de trabalho de enfermagem (TISS-28) antes e após o erro de medicação. Já o estudo dessas alterações segundo o grupo de medicamentos en- 
volvidos (MPP ou MNPP), antes e após a ocorrência, foi feito utilizando-se o teste de Análise de Variância (ANOVA) que avaliou simultaneamente os dois grupos de medicamentos e os dois momentos relacionados à ocorrência com medidas repetidas. Foram considerados estatisticamente significantes os resultados cujos níveis descritivos (valor de $p$ ) foram inferiores a 0,05 .

\section{RESULTADOS}

No período de quatro anos analisados nesta investigação, verificou-se que, dos 50 pacientes internados nas UTIs e USIs das instituições campo de estudo, 48 (96\%) sofreram um erro de medicação durante a permanência naquelas unidades. Dois pacientes (4\%), no entanto, sofreram dois erros cada um. Do total da amostra, 35 (70\%) pacientes encontravam-se nas UTIs e $15(30 \%)$ nas USIs.

A caracterização dos dados sócio demográficos mostrou a predominância de pacientes do sexo masculino (66\%). Quanto à procedência, 34\% foi proveniente do Pronto Socorro seguida das Unidades de Internação (28\%) e Centro Cirúrgico (22\%). Observou-se que a maioria dos doentes, (52\%) foi internada para tratamento clínico, prevalecendo as doenças do sistema cardiovascular (31\%) e respiratório (27\%) como motivos de internação. Após a alta das unidades, 38\% dos pacientes foram encaminhados para USI, $24 \%$ evoluíram à óbito e $20 \%$ obtiveram alta para as Unidades de Internação. Referente às doenças crônicas, $44 \%$ da população era portadora de uma e 36\% de nenhuma dessas doenças. No entanto, $20 \%$ da população possuía duas ou mais patologias crônicas associadas.

A idade média dos pacientes vítimas de erros de medicação nas UTIs e USIs foi de 62,32 anos (dp=18,87) com variação mínima de 17 e máxima de 90 anos, sendo o tempo médio de permanência nas unidades de 12,45 dias $(\mathrm{dp}=18,78)$, variando de um a 111 dias.

A análise dos resultados obtidos no período do estudo, permitiu constatar a ocorrência de um total de 52 erros de medicação nas UTIs e USIs, dos quais $37 \%$ e $35 \%$ aconteceram respectivamente nos turnos da tarde e manhã. Menor freqüência (29\%) foi encontrada no plantão noturno.

Observou-se além disso, que os erros de medicação ocorreram quando os pacientes tinham em média $4,53(\mathrm{dp}=5,86)$ dias de internação na UTI e USI, com variação de um a 25 dias.

Os dados da figura 1 mostram a distribuição dos erros de medicação segundo o tipo de evento.

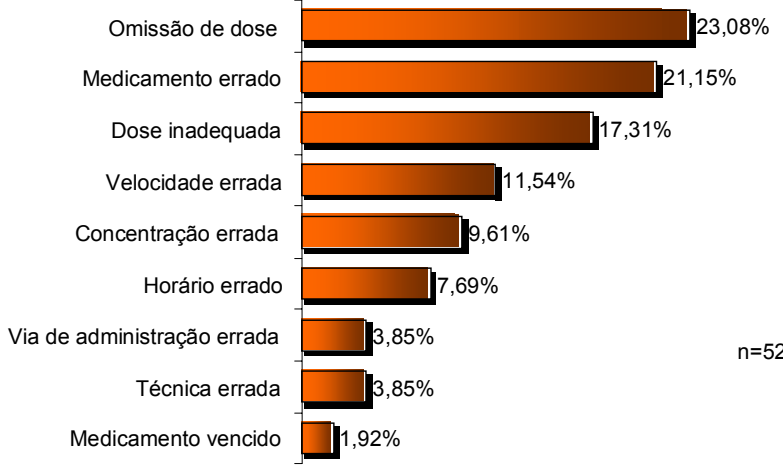

Figura 1 - Distribuição da porcentagem dos erros de medicação segundo o tipo de evento - São Paulo - 2004

Referente ao tipo de erro de medicação, nota-se na figura 1, que dos 52 erros encontrados, 12 (23,08\%) ocorreram por omissão de dose, seguidos de $11(21,15 \%)$ e $9(17,31 \%)$ por medicamento e dose errados, respectivamente. Freqüências menores foram relacionadas à velocidade errada $(6-11,54 \%)$, concentração errada (5 - 9,61\%) e horário errado (4-7,69\%), seguidas de via de administração e técnica incorretas com dois $(3,85 \%)$ cada um. Apenas um $(1,92 \%)$ erro foi relacionado à administração de medicamento vencido.

Quanto ao grupo de medicamentos envolvidos, a maioria dos erros $(59,60 \%)$ ocorreu com medicamentos não potencialmente perigosos (MNPP), enquanto que $40,40 \%$ envolveram o grupo de MPP.

Tabela 1 - Distribuição da média da pontuação SAPS II e TISS-28 antes e após a ocorrência de erro de medicação - São Paulo - 2004

\begin{tabular}{|c|c|c|c|}
\hline \multirow{2}{*}{ Índices } & \multicolumn{2}{|c|}{$\begin{array}{l}\text { Momentos } \\
\text { Média (dp) }\end{array}$} & \multirow{2}{*}{ p-valor } \\
\hline & Antes & Após & \\
\hline SAPS II & $44,27(13,86)$ & $42,62(13,64)$ & 0,316 \\
\hline TISS-28 & $24,40(9,04)$ & $26,54(8,98)$ & $0,009 *$ \\
\hline
\end{tabular}

* estatisticamente significante

Nota-se pelos dados da tabela 1 , que houve alteração estatisticamente significante ( $p$-valor $=0,009)$ na média de pontuação TISS-28 dos pacientes vítimas de erro de medicação, após 24 horas da ocorrência, indicando aumento da carga de trabalho de enfermagem. Por outro lado, não foi observada diferença estatisticamente significante (p-valor $=0,316$ ) na gravidade das condições clínicas, avaliada pelo SAPS II. 
Tabela 2 - Distribuição da média da pontuação SAPS II dos pacientes vítimas de erros de medicação segundo o grupo de medicamentos envolvidos e momentos relacionados à ocorrência - São Paulo - 2004

\begin{tabular}{lccc}
\hline Medicamentos & \multicolumn{2}{c}{$\begin{array}{c}\text { Momentos } \\
\text { Média (ep) }\end{array}$} & p-valor \\
& Antes & Após & \\
\hline MPP & $46,86(3,02)$ & $43,19(3,01)$ & 0,456 \\
MNPP & $42,52(2,48)$ & $42,23(2,47)$ & \\
\hline
\end{tabular}

ep = erro padrão

Conforme dados da Tabela 2, verifica-se que a gravidade dos pacientes, mensurada com o SAPS II, não apresentou diferença estatisticamente significante $(\mathrm{p}=0,456)$ após o erro de medicação, quando se considerou o grupo de medicamentos.

Os dados referentes à carga de trabalho de enfermagem após a ocorrência de erro de medicação segundo MPP e MNPP encontram-se a seguir.

Tabela 3 - Distribuição da média da pontuação TISS-28 dos pacientes vítimas de erros de medicação segundo o grupo de medicamentos envolvidos e momentos relacionados à ocorrência - São Paulo - 2004

\begin{tabular}{lccc}
\hline \multirow{2}{*}{ Medicamentos } & \multicolumn{2}{c}{$\begin{array}{c}\text { Momentos } \\
\text { Média (ep) }\end{array}$} & p-valor \\
& Antes & \multicolumn{1}{c}{ Após } & \\
\hline MPP & $26,14(1,97)$ & $28,05(1,96)$ & 0,264 \\
MNPP & $23,23(1,62)$ & $25,52(1,61)$ & \\
\hline
\end{tabular}

ep= erro padrão

Observa-se na tabela 3 que resultado semelhante ao do SAPS II ocorreu com relação ao TISS-28, ou seja, não houve mudança estatisticamente significante $(\mathrm{p}=0,264)$ na carga de trabalho de enfermagem após erro de medicação com MPP e MNPP.

\section{DISCUSSÃO}

Neste estudo, erros de medicação na prática assistencial em UTI e USI foram investigados com ênfase nas conseqüências acarretadas nas condições clínicas do paciente e na carga de trabalho de enfermagem.

A caracterização dos pacientes quanto ao gênero e idade, mostrou que a maioria é do sexo masculino (66\%) e idosa, com uma média de idade de 62,32 anos ( $\mathrm{dp}=18,27)$, dados compatíveis ao de estudos nacionais e internacionais ${ }^{(10,13-23)}$.

Sobre a procedência, a maioria (34\%) foi proveniente do Pronto Socorro. Diferente dos resultados deste estudo, autores diversos identificaram uma maior proporção de pacientes vindos do Centro Cirúrgico ${ }^{(13,18,20)}$. Entretanto, resultados próximos ao desta investigação foram encontrados em um estudo que avaliou a utilização do SAPS II em uma Unidade de Cuidados Intermediários de um hospital geral francês, onde, de um total de 433 pacientes, $60,9 \%$ procederam do Pronto Socorro, seguidos de 31,3\% das Unidades de Internação ${ }^{(10)}$.

As variações frente à literatura, podem ser justificadas pelo fato de a amostra considerar conjuntamente pacientes internados em UTI e USI. Além disso, também os hospitais, por apresentarem características diferentes, podem ter contribuído para essas variações.

Analisando-se o tipo de tratamento, observou-se que a maioria dos atendimentos foi clínico $(52 \%)$, provavelmente pelo fato de os pacientes procederem principalmente, neste estudo, do Pronto Socorro e Unidades de Internação, dados corroborados por estudo realizado em uma UTI adulto de um hospital do Município de São Paulo, onde, 55\% dos pacientes foram internados por razões clínicas ${ }^{(22)}$.

Os motivos que mais levaram os pacientes à internação nas UTIs e USIs foram as doenças do sistema cardiovascular (31\%) seguidos das doenças do sistema respiratório (27\%). Com relação a esses achados, também, o Segundo Censo Brasileiro de UTIs da Associação de Medicina Intensiva Brasileira (AMIB ${ }^{(24)}$ ), revelou que as quatro principais causas de internação foram as doenças do sistema cardiovascular, do sistema respiratório, sepse e pós operatório. Resultados semelhantes também foram encontrados em estudos nacionais e internacionais ${ }^{(17,20,25)}$, mostrando similaridade nas características dos pacientes graves, independente do país de origem.

Referente às condições de saída dos pacientes das unidades, constatou-se que $76 \%$ obtiveram alta e $24 \%$ evoluíram à óbito, resultados referendados em.várias UTIs brasileiras ${ }^{(13-14,22,26-27)}$. Observou-se, além disso, que dos $70 \%$ dos pacientes internados nas UTIs, 38\% foram encaminhados para as USIs, reforçando o papel dessas unidades na continuidade do tratamento intensivo.

A média de permanência de 12,45 dias, com variação de um dia a 111 dias que, embora elevada, encontra respaldo em estudo nacional que teve média de 8,32 dias com variação semelhante ${ }^{(22)}$. Apesar disso, não se pode desconsiderar que a análise conjunta dos dados das UTIs com as USIs pode ter sido responsável pela permanência elevada, sobretudo em função dessa última.

Considerando-se o tempo de internação até a ocorrência do erro de medicação, observou-se uma média de 4,53 dias, com variação do primeiro ao $25^{\circ}$ dia indicando a imprevisibilidade desses eventos. No entanto, estudo realizado em sete UTIs do Município de São Paulo sobre ocorrências iatrogênicas, verificou que de um total de 113 ocorrências, $46,9 \%$ se deram quando o paciente tinha até cinco dias de internação nessas unidades, resultado bastante próximo ao desse estudo ${ }^{(6)}$.

$\begin{aligned} \text { Rev Esc Enferm USP } & \text { Conseqüências dos erros de medicação em } \\ 2006 ; 40(2): 247-52 . & \text { unidades de terapia intensiva e semi-intensiva } \\ \text { www.ee.usp.br/reeusp/ } & \text { Toffoletto } M C, \text { Padilha KG. }\end{aligned}$


Referente ao horário em que ocorreram os erros de medicação, verificou-se uma distribuição muito próxima entre os períodos da tarde (37\%) e da manhã (35\%) com porcentagem menor à noite $(29 \%)$. As diferenças entre os períodos diurno e noturno talvez se justifiquem pelo excesso de atividades e maior número de profissionais presentes nas unidades no período diurno, acarretando ao profissional de enfermagem acúmulo de atividades com conseqüente cansaço e desatenção.

Considerando-se os erros de medicação quanto ao tipo, do total de 52 ocorrências, foram encontrados $23,08 \%$ erros por omissão de dose, seguidos de $21,15 \%$ e $17,31 \%$ por medicamento errado e dose inadequada, respectivamente. A grande variedade na tipologia dos erros econtrada na literatura nacional e internacional ${ }^{(6,13,28-29)}$, parece apenas refletir a complexidade inerente a essa atividade. Porém, independente da natureza, esses eventos devem ser analisados numa perspectiva multidimensional, tendo por base a premissa de que é o resultado de um processo que apresentou falha em alguma de suas fases.

Quanto aos grupos de medicamentos envolvidos nos erros, foi interessante observar que a maioria $(59,6 \%)$, pertencia à categoria de medicamentos não potencialmente perigosos (MNPP), contrariamente ao esperado nas UTIs e USIs. É possível supor que os MPP, em razão dos seus efeitos, sejam mais sujeitos à vigilância, o que evitaria maior ocorrência de erros. Apesar disso, a constatação de cerca de $40 \%$ de erros com essa categoria de medicamentos merece atenção e cuidados especiais dos enfermeiros no sentido da sua prevenção.

O aprofundamento da análise das conseqüências dos erros de medicação, com enfoque no grupo de medicamentos envolvidos, mostrou que não houve mudança na gravidade dos pacientes e na carga de trabalho de enfermagem ( $\mathrm{p}=0,456 \mathrm{e} \mathrm{p}=0,264$, respectivamente), após a ocorrência do erro. Ao mostrar que as intervenções de enfermagem foram iguais, os resultados deixam evidente que a responsabilidade dos profissionais na monitorização e controle dos pacientes prevaleceu independente do grupo de medicamentos envolvidos.

Neste estudo, também foi inesperado verificar que a gravidade dos pacientes não se alterou após o erro de medicação ( $\mathrm{p}=0,316)$, havendo, porém, aumento da carga de trabalho de enfermagem $(\mathrm{p}=0,009)$.

Tais dados vem demonstrar que, as conseqüências dos erros de medicação recaíram principalmente sobre a equipe de enfermagem que teve sua carga de trabalho de enfermagem aumentada, certamente, pela intensificação da vigilância e intervenções programadas no intuito de evoluir as condições clínicas dos pacientes, após a ocorrência, e identificar precocemente sinais indicativos de complicações.

\section{CONSIDERAÇÕES FINAIS}

Frente a elevada quantidade de medicamentos preparados e administrados diariamente nas UTIs e USIs, o pequeno número de erros de medicação encontrados em um período de quatro anos, remete a algumas considerações. A despeito da qualidade dos cuidados prestados nas instituições campo do estudo, que pressupõem uma baixa ocorrência de erros, não se pode desconsiderar as subnotificações, decorrentes não só de uma cultura de punição, como também do medo das sanções ético-legais possíveis.

Apesar disso, nos últimos anos, investimentos têm sido feitos para estimular as notificações, alavancados pelos programas de qualidade dos serviços de saúde e acreditação hospitalar que, ao considerarem os erros de medicação como indicadores de resultado, têm contribuído de forma expressiva para um novo enfoque a esse tipo de evento.

Porém, embora se tenha avançado em direção à monitorização dos erros de medicação e à implantação de sistemas de registros de ocorrências, inclusive por meio de programas oficiais de gerenciamento de riscos nas instituições hospitalares, a realidade mostra a necessidade de engajamentos mais efetivos.

A constatação neste estudo de que a maioria dos pacientes foi vítima de apenas um erro de medicação, não evita preocupação quando se busca uma assistência livre de erros, além do que, um único erro pode ser suficiente para desencadear conseqüências indesejáveis. Nesse sentido, torna-se fundamental a valorização desses eventos, não apenas pela significância quantitativa, mas, sobretudo, pela dimensão das suas conseqüências para o paciente grave, objeto de estudo nesta investigação.

Deparar-se na literatura com estudos que abordam as conseqüências decorrentes de erros de medicação com o uso de diferentes metodologia, na sua maioria baseadas na observação e nos relatos vividos pelos profissionais, dificultou a comparação dos resultados desta investigação. É certo, porém, que existe carência de estudos que focalizem objetivamente as conseqüências dos erros de medicação nas condições clínicas do paciente grave e na carga de trabalho da equipe de enfermagem com a aplicação de escalas de medida objetivas. Tais instrumentos tornam-se vantajosos por complementarem as análises subjetivas contidas em relatos feitos pelos profissionais.

Em síntese, em que pese a relevância dos resultados deste estudo, problemas metodológicos, sobretudo relacionados ao tamanho da amostra, trazem limitações para a generalização dos dados. Contudo, apontam para a necessidade de um maior número de estudos que investiguem as conseqüências dos erros de medicação com a utilização de instrumentos de medidas objetivos, com vistas a verticalizar o conhecimento sobre tema tão complexo. 
(1) Padilha KG. Iatrogenia em enfermagem na Unidade de Terapia Intensiva: análise multidimensional do problema. Rev Esc Enferm USP. 1998;22(1):141-3.

(2) Padilha KG. Iatrogenias em Unidade de Terapia Intensiva: uma abordagem teórica. Rev Paul Enferm. 1992;11(2):69-72.

(3) Padilha KG, Secoli SR. Erros na administração de medicamentos. Prat Hosp. 2002;4(19):24-9.

(4) Padilha KG. Ocorrências iatrogênicas na UTI e o enfoque de qualidade. Rev Lat Am Enferm. 2001;9(5):91-6.

(5) Padilha KG. A prática de enfermagem em UTI e as ocorrências iatrogênicas: considerações sobre o contexto atual. Rev Paul Enferm. 2000;19(3):49-56.

(6) Padilha KG. Ocorrências iatrogênicas na prática de enfermagem em UTI. [tese livre-docência]. São Paulo: Escola de Enfermagem da USP; 1998.

(7) Livianu J, Anção MS, Akamine N, Andrei AM. Índices prognósticos em UTI. In: Knobel E, Kuhl SD. Condutas no paciente crítico. São Paulo: Atheneu; 1994. Cap. 67, p. 823-33.

(8) Miranda DR, Rijk A, Schaufeli W. Simplified Therapeutic Intervention Scoring System: the TISS-28 items results from a multicenter study. Crit Care Med. 1996;24(1):64-73.

(9) Cullen DJ, Civetta JM, Briggs BA, Ferraro LC. Therapeutic Intervention Scoring System: a method for quantitative comparison of patient care. Crit Care Med. 1974;2(2):57-60.

(10) Auriant I, Vinatier I, Thaler F, Tourneur M, Loirat P. Simplified Acute Physiology Score II for measuring severity of illness in intermediat care units. Crit Care Med. 1998;26(8):1368-71.

(11) Porath A, Reuveni H, Grinberg G, Lieberman D. The intermediate care unit as a cost-effective option for the treatment of medical patients in critical condition. Isr J Med Sci. 1995;31(11):674-80.

(12) Zimmerman JE, Wagner DP, Sun X, Knaus WA, Draper EA. Planning patient services for intermediate care units: insights based on care for intensive care unit low-risk monitor admissions. Crit Care Med. 1996; 24(10):1626-32.

(13) Silva SC. Ocorrências iatrogênicas em unidade de terapia intensiva: impacto na gravidade e na carga de trabalho de enfermagem [tese]. São Paulo: Escola de Enfermagem da USP; 2003.

(14) Nascimento EFA. Evolução da gravidade de pacientes adultos internados em uma Unidade de Terapia Intensiva [dissertação]. São Paulo: Escola de Enfermagem da USP; 2002.

(15) Nunes B. Tradução para o português e validação de um instrumento de medida de gravidade em UTI: TISS-28 [tese]. São Paulo: Escola de Enfermagem da USP; 2000.

(16) Paiva SAR, Matai O, Resende NO, Campana AO. Análise de uma população de doentes atendidos em Unidade de Terapia Intensiva - estudo observacional de sete anos (1992-1999). Rev Bras Ter Intensiva. 2002;14(2):173-80.
(17) Factore LAP. Indicadores e prognósticos em pacientes graves [tese]. São Paulo: Faculdade de Medicina da USP; 2000.

(18) Bastos PG, Sun X, Wagner DP, Knaus WA, Zimmerman JE. Application of the Apache III prognostic system in Brazilian Intensive Care Units: a prospective multicenter study. Intensive Care Med. 1996;22(6):564-70.

(19) Castillo EL, Rivera FR, Rodrigues EM, Vasquez MG. TISS76 and TISS-28: correlation of two therapeutic activity indices on a Spanish multicenter ICU database. Intensive Care Med. 2000;26(1):57-61.

(20) Silva MCM, Sousa RMC. Caracterização dos pacientes adultos e adolescentes das Unidades de Terapia Intensiva do Município de São Paulo. Rev Paul Enferm. 2002;21(1):50-7.

(21) Metnitz PG, Vesely H, Valentin A, Popow C, Hiesmayr M, Lenz K, et al. Evaluation of an interdisciplinary data set for national intensive care unit assessment. Crit Care Med. 1999;27(8):1486-91.

(22) Telles SCR. Custo de pessoal na assistência direta de enfermagem em Unidade de terapia Intensiva [dissertação]. São Paulo: Escola de Enfermagem da USP; 2003.

(23) Ducci AJ, Padilha KG, Telles SC, Gutierrez BAO. Gravidade de pacientes e demanda de trabalho de enfermagem em Unidade de Terapia Intensiva: análise evolutiva segundo o TISS-28. Rev Bras Ter Intensiva. 2004;16(1):22-7.

(24) Associação de Medicina Intensiva Brasileira. $2^{\circ}$ Anuário Brasileiro de UTIs. São Paulo; 2002-2003.

(25) Miranda DR, Ryan DW, Schaufeli WB, Fidler V. Organization and management of intensive care: a prospective study in 12 European Country. Berlin: Springer; 1998.

(26) Nogueira GP. Indicadores de gravidade em Unidade de Terapia Intensiva [dissertação]. São Paulo: Escola de Enfermagem da USP; 2002

(27) Tranquitelli AM. Estudo prospectivo para determinação do número de horas de cuidados diretos de enfermagem em uma Unidade de Terapia Intensiva Geral [dissertação]. São Paulo: Escola de Enfermagem da USP; 1999.

(28) Van den Bemt PMLA, Fijn R, Van der Voort PHJ, Gossen AA, Egberts TCG, Brouwers JR. Frequency and determinants of drug administration errors in the intensive care unit. Crit Care Med. 2002;30(4):846-50.

(29) Schneider MP, Cotting J, Pannatier A Evaluation of nurses' errors associated in the preparation and administration of medication in a pediatric intensive care unit. Pharm World Sci. 1998;20(4):178-82. 


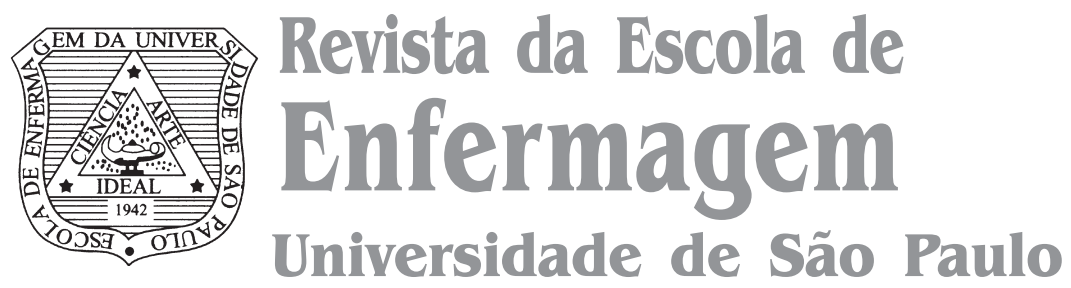

ERRATA

Como publicado no volume 40, número 2 de 2006, página 247, onde lê-se "Conseqüências de medicação em unidades de terapia intensiva e semi-intensiva", leia-se: "Conseqüências dos erros de medicação em unidades de terapia intensiva e semi-intensiva".

A citação correta em referência é:

Toffoletto MC, Padilha KG. Conseqüências dos erros de medicação em unidades de terapia intensiva e semi-intensiva. Rev Esc Enferm USP. 2005;40(2):247-52. 
O arquivo disponível sofreu correções conforme ERRATA publicada no Volume 40 Número 3 da revista. 